\title{
Terahertz Imaging to Map the Microporosity Distribution in Carbonate Rocks
}

Shannon Eichmann ${ }^{1}$, Jacob Bouchard ${ }^{2}$, Hooisweng Ow ${ }^{3}$, Patrick O'Mullan ${ }^{4}$, Doug Petkie ${ }^{4}$ and Martin Poitzsch ${ }^{3}$

${ }^{1}$ Aramco Services Company: Aramco Research Center - Houston, Houston, Texas, United States, ${ }^{2}$ Department of Physics, Worcester Polytechnic Institute, Bellingham, Massachusetts, United States, ${ }^{3}$ Aramco Services Company: Aramco Research Center - Boston, United States, ${ }^{4}$ Department of Physics, Worcester Polytechnic Institute, United States

Conventional oil reservoirs are mainly comprised of either sandstone or carbonate minerals and pores that contain hydrocarbons and water. Approximately $50 \%$ or more of the oil reserves worldwide are stored in carbonate reservoirs [1]. The porosity $(\phi)$ and pore size distribution (PSD) of reservoir rocks affects hydrocarbon storage and transport and thus the producibility of these reservoirs. While the majority of pores in sandstones are on the order of $10 \mathrm{~s}$ of microns in diameter, carbonate rocks can have a significant micro porosity $\left(\phi_{\mu}, \mathrm{d}_{\text {pore }}<1 \mu \mathrm{m}\right)$. Many techniques exist to measure the $\phi$ and PSD of reservoir rocks. Mercury Injection Capillary Pressure (MICP) is commonly used to quantify the $\phi$ and PSD but mercury destroys the sample and its use is undesirable due to health and safety concerns. Gas adsorption also provides $\phi$ and PSD but neither this nor MICP show the spatial distribution of the $\phi$. Imaging techniques such as microCT and optical microscopy are also used to measure $\phi$ and PSD but many of the micropores in carbonate rocks are too small to image due to resolution limitations.

Terahertz (THz) spectroscopy is a non-destructive tool used in many industries to analyze materials. THz TimeDomain Spectroscopy measures the amplitude and phase of the electric field of a THz pulse. This pulse is sensitive to dielectric and structural properties that vary as a function of frequency. In the biological and environmental communities, the attenuation of the $\mathrm{THz}$ signal due to water has been used to detect and map the moisture content in animal tissues and plants [2-4]. Previous work with limestone samples demonstrated that the $\mathrm{THz}$ signal is significantly attenuated due to water-filled pores in saturated samples and that the attenuation decreases as the water content is reduced by dehydration in a nitrogen environment [5]. Thus, with increasing drying time the $\mathrm{THz}$ attenuation is linked to the decrease in filled $\phi$ and the fully saturated $\phi$ to the effective (i.e. connected) $\phi$ [5]. This study additionally showed that the THz attenuation versus time could be correlated to the amount of $\phi_{\mu}$ [5]. Two drying rates that were used to identify the $\mathrm{THz}$ attenuation due to drying of macropores and micropores and then calculate the percentage of each, which agreed with MICP measurements [5]. Drying samples in air, however, is a slow process and the drying rate transition from larger pores to drying smaller pores is not based on a pore size cutoff. In addition, a single-point measurement does not provide information about how the $\phi_{\mu}$ spatial distribution.

Here we extend the previous work using THz imaging to quickly map lateral variations in $\phi_{\mu}$ using the attenuation due to water in the pores after selectively clearing the large pores via centrifugation. Carbonate rock samples were selected with differing effective $\phi$. Each core plug is cut into circular discs of varying thickness, which are cut in half to produce half-moon shaped samples. The remainder of the core plug is used for MICP measurements. First, each sample is weighed then saturated with deionized water under vacuum to imbibe water and displace air. The saturated samples are weighed and imaged using a $\mathrm{THz}$ imaging system where the water filling the pores significantly attenuates the $\mathrm{THz}$ signal. Next, centrifugation selectively clears water from the pores larger than a desired threshold diameter, using capillary pressure effects $[6,7]$. After centrifugation the samples are weighed and imaged to map the remaining water-filled $\phi_{\mu}$. Finally, the samples are dried in an oven overnight, weighed, and imaged for a baseline image without water. The imaging parameters were consistent for all imaging steps. The system uses a raster scanning computer-controlled imaging gantry to produce images $19 \mathrm{~mm}$ wide by $42 \mathrm{~mm}$ tall at $0.5 \mathrm{~mm} / \mathrm{step}$. A reference pulse was collected in the imaging window without a sample present for calibration.

Results are presented that compare $\mathrm{THz}$ attenuation maps to effective $\phi$ and $\phi \mu$ estimations from mass balance and MICP. The $\phi$ of the THz samples is measured from the mass difference between the dry and saturated water states and compared to the $\phi$ from the whole plug. On average, the $\phi$ is $\sim 20 \%$ higher than the independent measurement which is not unreasonable given the smaller sample size and the effect of subsampling on pore connectivity. The mass difference between the centrifuged and dried sample was used to estimate the $\phi_{\mu}$ which ranges from $20 \%$ to $30 \%$ of the total $\phi$. The estimates of $\phi_{\mu}$ are compared to that expected from independent measurements from MICP. 
THz attenuation maps (Figure 1) are aligned and compared at each saturation step: saturated, centrifuged, and dry. The images show increased average transmission between steps and consistent distributions within the sample. To ensure that the changes observed in discrete regions were due to higher $\phi_{\mu}$, the percent change of the average transmitted electric field through whole sample was compared with that for certain regions of interest (ROIs). The average transmitted electric field through the whole sample increased by $76 \%$ from the saturated step to the centrifuged step while the average within the ROI (red box) only increased by 56\%. Between the centrifuged step and the dried step, the average electric field through the sample increased by $46 \%$ while the average in the ROI (red box) increased by $70 \%$. This demonstrates that some regions contain more than the average amount of water after centrifugation suggesting that they contain a higher than average amount of micropores. Next steps include repeating the measurements to selectively clear pores above a smaller threshold pore size to confirm attenuation map observations and improving data analysis to account for the impact of $\mathrm{THz}$ scattering and absorption in the dried regions. These results demonstrate the use of $\mathrm{THz}$ imaging to quantify and map $\phi_{\mu}$ in carbonate reservoir rocks which may provide a new detection method for core analysis.
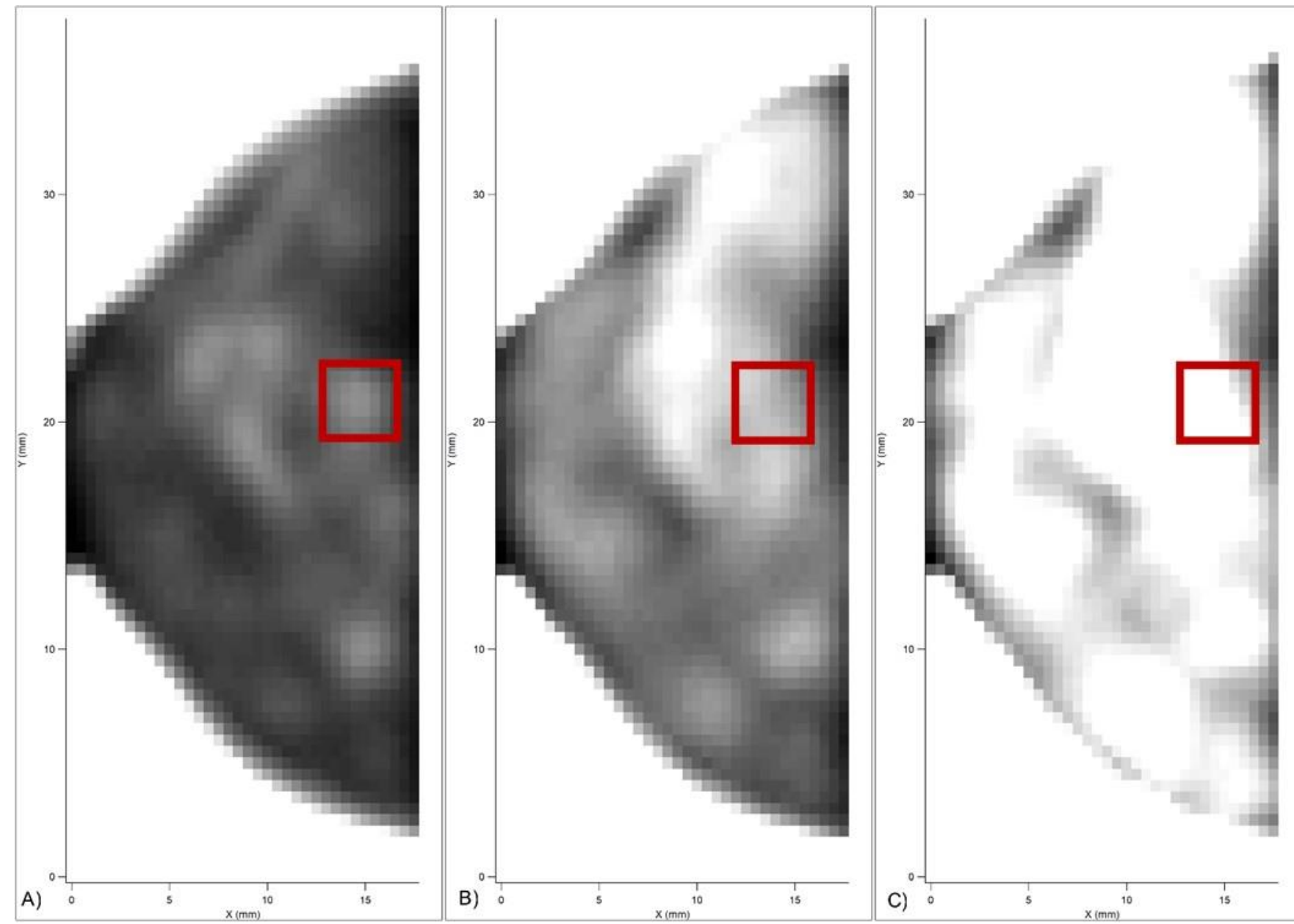

Figure 1. Figure 1: THz electric field intensity images of a $4 \mathrm{~mm}$ thick limestone sample A) saturated B) after centrifugation and C) after oven drying. The images were produced by taking the peak to peak amplitude of the $\mathrm{THz}$ pulse for each pixel and a consistent linear color scale was applied to each image to prevent high signal areas around the sample from skewing the scale too high and hiding internal features. The red square illustrates an region of interest suspected of having a high density of micropores.

\section{References}

[1] Roehl, P. O., \&Choquette, P. W. (Eds.). (2012). Carbonate petroleum reservoirs. Springer Science \& Business Media. 
[2] Banerjee, D., et al. Optics Express 16.12 (2008).

[3] Wang, Yuye, et al. Optics Express 18.15 (2010).

[4] Castro-Camus, E., et. al. Scientific Reports 3.1 (2013).

[5] Heshmat, B., et al. Optics Express 25.22 (2017).

[6] Howard, J. J., et al. The Log Analyst 36.01 (1995).

[7] Dandekar, A. Y. (2013). Petroleum reservoir rock and fluid properties. CRC press. 